\title{
Interfacial Adhesion due to Liquid Mediated Contact of Hydrophilic Solid Surfaces
}

\author{
Shaobiao Cai and Prabin Dhital \\ Mechanical Engineering \\ Minnesota State University \\ Mankato, USA
}

\author{
Yongli Zhao \\ Mechanical and Manufacturing Engineering \\ St. Cloud State University \\ St. Cloud, USA
}

\begin{abstract}
When liquid presents between solid contact hydrophilic surfaces, the formed macro/micro menisci cause inherit adhesive force due to surface tension. Such adhesive force may lead high friction and adhesion and cause system failure of many mechanical systems, and thus liquid caused high adhesion is one of the major limit factors to many micro/nano devices having contact surfaces with relative motion. In this study, the involved forces such as meniscus force were modeled, separation processes were numerical simulated and analyzed. Various liquids, such as water and commonly used liquid lubricants, were used. Informative results and insights of the separation of hydrophilic solid surfaces from liquid were obtained and presented.
\end{abstract}

Keywords-Meniscus force; solid-liquid interaction; adhesion; viscous force

\section{INTRODUCTION}

When liquid presents at the contact interface of two surfaces, menisci may form around the contacting and near contacting asperities due to surface energy effect of a thin liquid film as shown in Fig. 1. When liquid mediated contact is considered, the formed menisci are the center of interest. The geometric description of the meniscus is the meniscus curve. It forms between the upper and lower surfaces of the liquid when the two surface are brought into contact. The shape of the meniscus is determined by the liquid and solid properties. In some cases, the liquid spreads evenly on the surface, and sometimes it forms into tiny droplets. Liquid spreading evenly on the surface with maximizing contact angle is known as hydrophilic phenomenon and liquid causing droplet is known as hydrophobic phenomenon. The meniscus is convex for a hydrophobic surface, and it is concave for a hydrophilic surface. Attractive force (meniscus force) acts on the interference for hydrophilic surfaces in contact and repulsive meniscus force for hydrophobic surfaces [1-3]. The angles formed between the meniscus curve and the contact surfaces are called contact angles as shown in Fig. 2. The contact angel can be measured through the liquid where a liquid/gas interface meets the solid surface with Sessile drop technique. The contact angle is an important indicator of the contacting system. For a multi-phase solid, such as liquid and gas system under certain conditions (i.e. different pressures and temperatures), the equilibrium of the system (represented by the unique contact angles) reflects the strength or energy level of the materials. The formation of the menisci around the contacting and near contacting surface asperities are due to the effect of the surface energy of the liquid film.
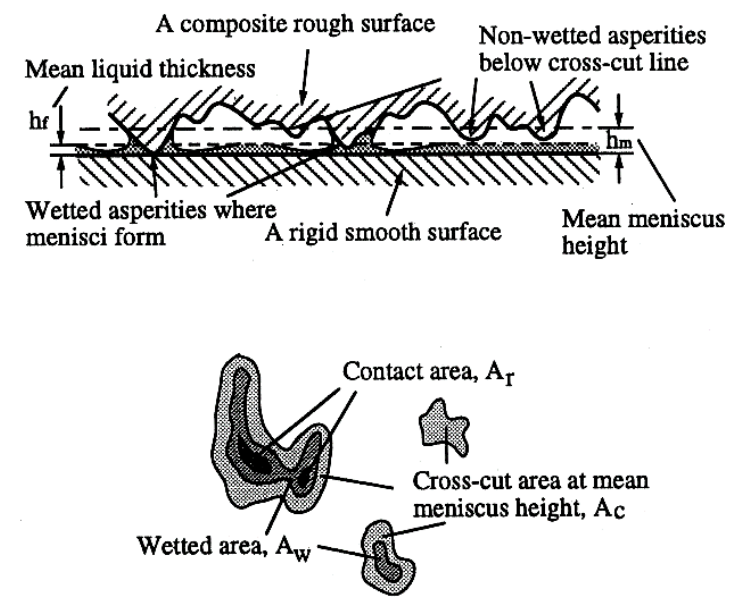

Projected meniscus area, $A_{m}=A_{c}^{*}-A_{r}$ $A_{C}^{*}$ is the cross-cut area of those islands which contain wetted area

Fig. 1. Schematics of menisci formed between liquid mediated contact $[2,3]$

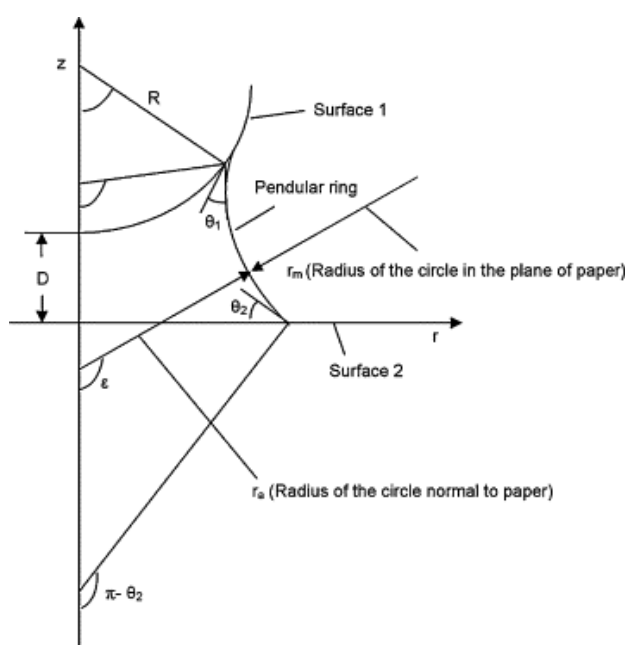

Fig. 2. Schematics of the meniscus curvature and contact angles at the contact interface of liquid mediated sphere-on-flat contact [4] 
When separate two liquid mediated contacting surfaces, meniscus and viscous forces have been believed to be the major contributors to the adhesion involved in the process. Many studies have been carried out to study the separation of liquid mediated contact. Cai and Bushan [1-3] developed models (CB model) and studied the separation of two flat-onflat surfaces and sphere-on-flat surfaces. In the CB model, various design parameters, such as contact angle, initial separation height, surface tension and liquid viscosity, have been investigated. More importantly, it has been found that the role of the involved meniscus and viscose forces changes when a so called critical meniscus area is reached.

Various factors need to be considered in order to characterize the involved forces for separating two liquid mediated surfaces. The effects of separation distance, initial meniscus height, separation time, and roughness have been studied by S. Cai and B. Bhushan [2, 3]. As one of the major factors in liquid mediated contact, the contact angle, has been studied intensively. The study on adhesion contributed by meniscus and viscous forces during the separation of two hydrophilic smooth surfaces with symmetric and asymmetric contact angles were carried out. In their study, a critical meniscus area was first identified and defined as the meniscus area at which the meniscus and viscous forces change role. It was found that the increasing in separation distance led to the meniscus forces to decrease but the integrative viscous force (needed to be overcome) to increase. The increase in both initial meniscus height (for rough surfaces) and surface roughness led to an increasing in meniscus force, but it did not affect the viscous force significantly [3].

Meniscus force contributes to an intrinsic adhesion due to the formed menisci. During the separation of contacting surfaces, the viscosity of the liquid causes an additional attractive force (a rate dependent viscous force). Both meniscus and viscous forces cause to an adhesive force during the separation. During the surface contact and when separation operation of two contact surfaces is needed, adhesion due to the meniscus and viscous force is one of the major reliability issues. The issue becomes severer when the applied load is small (which is common) for micro or nanoparticle devices. The force required separating two surfaces dependent on the total force of both meniscus and viscous forces. It has been found that this type of adhesive force is highly dependent on the formed meniscus area, separation time, and surface tension and viscosity of the liquid. Since friction and wear are closely connected with the phenomenon of adhesion so the role of adhesion is important and we can take advantages of it with insight understanding in many applications [5].

In this paper, the critical meniscus area at which meniscus and viscous force changing roles are characterized. The factors of liquid properties (such as surface tension and viscosity), initiate surface separation distance (meniscus height) etc. are studies.

\section{MODELING OF THE FORCES}

To study the separation of liquid mediated contact from the liquid meniscus, a flat-on-flat model (as shown in Fig. 3) is used.

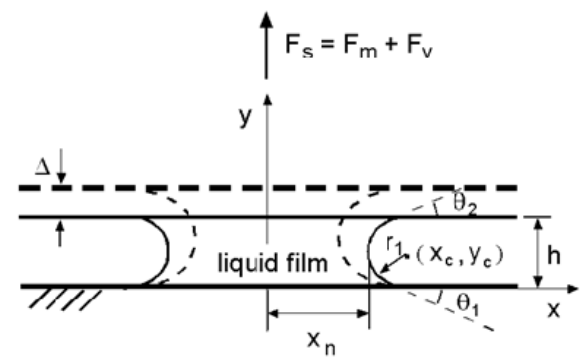

Fig. 3. Schematic of separation of flat-on-flat from a liquid meniscus

\section{A. Meniscus force}

First, Meniscus forming between the two flat surfaces due to a surface tension $\gamma$ resulting in pressure difference $\Delta p$ (which is often referred to as capillary pressure or Laplace pressure) is given by the Laplace equation [6].

$$
\Delta p=\gamma\left(\frac{1}{r_{1}}+\frac{1}{r_{2}}\right)
$$

where, $r_{1}$ is the meniscus radius as shown in Fig. 1a, and $r_{2}$ is another radius of the meniscus in the orthogonal plane to the plane $r_{1}$ belonging to (not shown in Fig. 3a).

It is noticed that one may neglect the surface tension contribution when the initial meniscus neck radius $x_{0}>>r_{1}$ is true. If $r_{2}>r_{1}$ does not hold, $r_{2}$ may be replaced with the difference between $x_{c}$, the center coordinate of the meniscus curve, and $\mathrm{r}_{1}$. Thus, $r_{2} \rightarrow x_{c}-r_{1}$ [7].

The meniscus height $h$ can be calculated based on the geometric configurations as shown in Fig. 3 for flat-on-flat case [8],

$$
h=r_{k}\left(\cos \theta_{1}+\cos \theta_{2}\right)
$$

where $r_{k}$ is the so called Kelvin radius and can be obtained from Kelvin equation using thermodynamics law. Thus, meniscus height is related to meniscus force. For a circular meniscus [1],

$$
F_{m}=\frac{\pi x_{n}^{2} \gamma\left(\cos \theta_{1}+\cos \theta_{2}\right)}{h}+2 \pi \gamma x_{n} \sin \theta_{1,2}
$$

where $\mathrm{x}_{\mathrm{n}}$ is the meniscus neck radius

$$
\mathrm{x}_{\mathrm{n}}=\mathrm{x}_{\mathrm{c}}-\mathrm{r}_{1} \sin \theta_{1,2}
$$

and it varies during separation. 


\section{B. Viscous Force}

Viscous force occurs due to the viscosity of the liquid when separating two bodies within a short time. During the separation, The pressure inside the meniscus bridge consists of horizontal pressure gradients, whereas the pressure is assumed constant in any vertical plane inside a meniscus bridge, and at the outside of a meniscus ring $\mathrm{r}=\mathrm{r}_{\mathrm{b}}$ (liquid-air interfacial boundary, which is exposed in ambient), the pressure $p\left(r_{b}\right)=p_{b}$, the ambient pressure.

Based on Reynolds' lubrication equation with cylindrical coordinate system [9]

$$
\frac{\partial}{\partial r}\left(r h^{3} \frac{\partial p}{\partial r}\right)=12 \eta r \frac{\partial h}{\partial t}
$$

The pressure difference in and outside the meniscus can be found by integrating the lubrication equation.

$$
\Delta p=\frac{3 \eta}{h^{3}}\left(r^{2}-x_{n}^{2}\right) \frac{\partial h}{\partial t}
$$

The average pressure difference between the center and the outside boundary of the meniscus which can be found as

$$
\Delta p_{\text {avg }}=-\frac{3 \eta}{2 h^{3}} x_{n}^{2} \frac{\partial h}{\partial t}
$$

Thus, the viscous force can be calculated by using the average pressure difference [1].

$$
F_{V}=\frac{3 \pi \eta x_{n}^{4}}{4 t_{s}}\left(\frac{1}{h_{s}^{2}}-\frac{1}{h_{0}^{2}}\right)
$$

where $t_{s}$ is the time to separate two bodies, and $h_{s}$ the break point and $h_{0}$ the initial gap between the two flat surfaces.

\section{Curvature of the meniscus During Separation}

During the separation of contact surfaces, it is assumed that the meniscus curvature remains circular since the separation time is small which is the case for many devices. The actual viscous force can drop very fast if the time to separate is long. Thus the effects of thermal and gravitation interference are believed to be negligible.

For arc shaped meniscus, the shape function may be expressed as

$$
\left(x-x_{c}\right)^{2}+\left(y_{m}-y_{c}\right)^{2}=r^{2}
$$

During the separation, the boundary conditions showed in Fig. 3 are implemented and conservation of the liquid volume is satisfied with the assumptions. The meniscus force at an instant simulation step depends on the corresponding instant curvature, which relies on the instant values of $x_{n i}, r_{i}$, and the center coordinate $x_{c i}$ of a meniscus while separating. These values can be found by using the meniscus profile with given initial conditions and boundary conditions.

\section{RESULTS AND DISCUSSION}

In the simulation, two flat surfaces were separated from a liquid meniscus. The meniscus curvatures are generated based on an initial $h_{0}=2 \mathrm{~nm}$ for the case of separating two flat surfaces. An initial meniscus radius of $100 \mathrm{~nm}$ is used. meniscus and viscous forces were calculated during separation based on various initial meniscus heights from $2 \mathrm{~nm}$ to $6 \mathrm{~nm}$. Meniscus heights from $2 \mu \mathrm{m}$ to $6 \mu \mathrm{m}$ are also used to calculate the critical meniscus areas (at which meniscus and viscous forces may dominate each other). The critical area as a function of initial liquid thicknesses (meniscus height) was identified.

Fig. 4 shows an example of instant meniscus curvatures during separating two parallel flat surfaces. Meniscus and viscous forces are calculated based on the instant curvatures during separation. Supposing meniscus breaks at zero meniscus neck radius. Thus at the break point, the forces disappear. The force needed to overcome a meniscus force is the meniscus force occurred at the initial point, whereas the force needed to overcome a viscous force (due to viscosity) equals the force resulting from the break of a meniscus bridge (which occurs at the break point).

The effects of separation distance to the meniscus and viscous forces can be observed in Figs. 5. Both meniscus force and viscous force exhibit a rapidly changing rate at the beginning. The larger rate change in the force at the beginning of separation is due to the larger rate change in volume at the beginning and becomes gradual thereafter (Fig. 4), and a larger volume change rate leads to a relatively larger decrease of the pressure difference (separation at a constant speed). It is also noticed that meniscus force decreases with separation distance, whereas viscous force has an opposite trend. This indicates that either of the two forces can be dominant during separation.

It is noticed that a larger meniscus area leads to larger meniscus and viscous forces. This can be easily observed from the results.

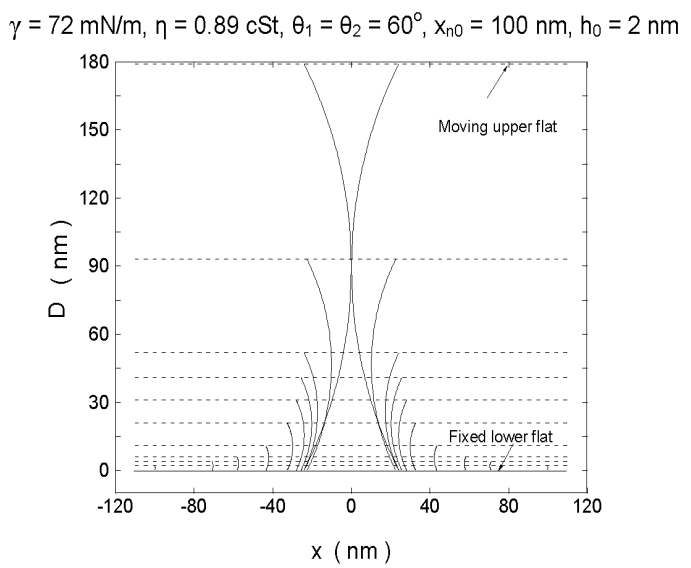

Fig. 4. Meniscus curvature during separation 
Fig. 5 below shows also both meniscus force and viscous force versus meniscus area, and the viscous force was calculated at a separation time $0.1 \mu \mathrm{s}$. The results show that the increase in meniscus area leads to large magnitude increase of the two forces. It is observed that viscous force is even more sensitive to meniscus area. The increase in viscous force with meniscus area is much faster than meniscus force does, and the opposite is also true. At a fixed separation time $t_{s}$, the two types of forces are comparable when a critical meniscus area is reached.

$$
\gamma=72 \mathrm{mN} / \mathrm{m}, \eta=0.89 \mathrm{cSt}, \mathrm{t}_{\mathrm{s}}=0.1 \mu \mathrm{s}
$$
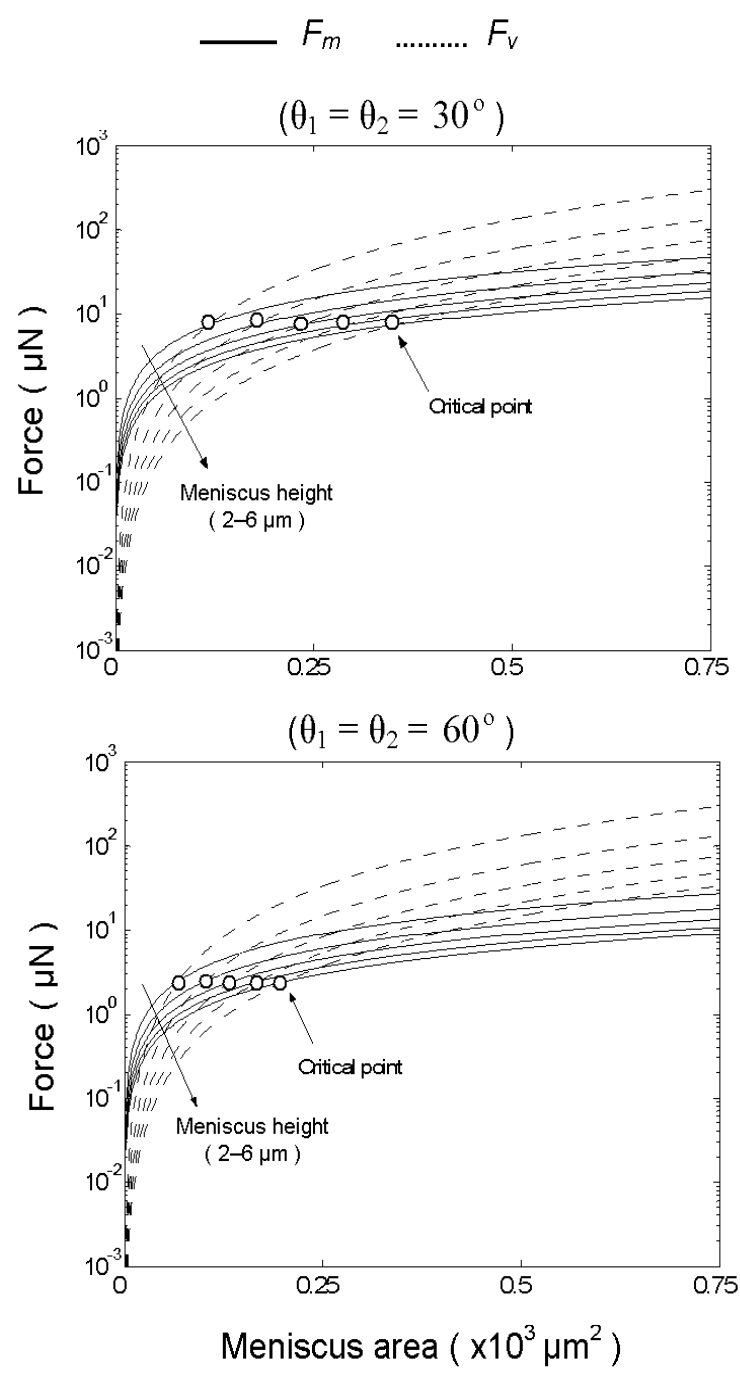

Fig. 5. Meniscus and viscous forces during separation for various initial meniscus heights and contact angles.

A continuous increase in meniscus area will result in much higher viscous force (as compared to meniscus force). These trends indicate that either of the two forces can be dominant dependent on the size of the meniscus area. The roles of the two forces largely depend on the size of the meniscus. Viscous force is dominate for a meniscus size larger than the critical one, and vice versa. Thus, one effective way to minimize the two forces is to minimize the meniscus area. One may neglect viscous force when meniscus area is small enough for a given separation time. But on the contrary, neglecting a viscous force for a big meniscus may lead to an underestimation of a total force needed to separate the two surfaces from liquid mediated contacts.

For the case of separating two parallel flat surfaces, it is shown that a lower meniscus height leads to a larger meniscus and viscous forces, and the increase in $h_{0}$ leads to a decrease of the two forces. This is because, at a fixed meniscus area, a higher $h_{0}$ results in a larger Kelvin radius and lower pressure difference $\Delta p$, and thus, corresponds to lower forces.

The results also show that both forces increase with the increase in meniscus area. Whereas, the one with smaller initial meniscus height increases much faster as compared to the others having larger meniscus heights. The initial meniscus height affects the critical area as well. At a given separation time $t_{s}$, a larger initial meniscus height corresponds to a larger critical meniscus area at which both forces are comparable. This is because a larger initial meniscus height leads to a much faster decrease of viscous force as compared to meniscus force, thus a larger meniscus area is needed for the viscous force to become comparable to meniscus force. Also, since the increase in the meniscus area and the increase in meniscus height lead to opposite effects to the change of forces, no surprising to observe only trivial changes in forces if both increase simultaneously.

Critical meniscus area as a function of initial separation heights from $2 \mathrm{~nm}$ to $6 \mathrm{~nm}$, and liquid surface tension and viscosity are presented in Fig. 6 and Fig. 7. For the calculation, the initial liquid properties were taken from silicon oil, $\gamma_{\mathrm{o}}=0.0633 \mathrm{~N} / \mathrm{m}$ and $\eta_{\mathrm{o}}=0.4860 \mathrm{Ns} / \mathrm{m}^{2}$.

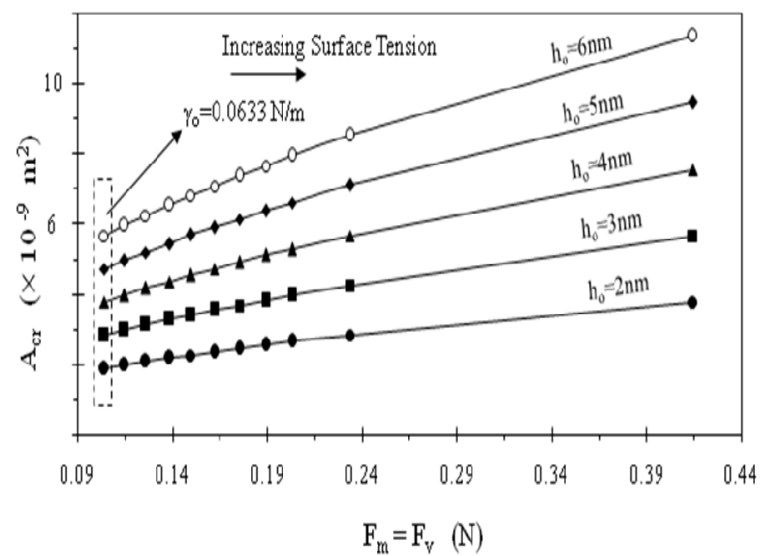

Fig. 6. Relationship between meniscus force and critical meniscus area with contact angles $\Theta_{1}=\Theta_{2}=60^{\circ}$, constant $\eta\left(0.4860 \mathrm{~N}-\mathrm{s} / \mathrm{m}^{2}\right)$ for separation time $=1 \mathrm{~s}$, Note: $\gamma_{\mathrm{o}}=0.0633 \mathrm{~N} / \mathrm{m}$ (Silicon Oil).

Fig. 6 shows the effects of initial meniscus height and surface tension to the critical meniscus area in a dimensional analysis. It is observed that for a constant initial meniscus height, the critical meniscus area (at which meniscus force equals the viscous force) increases with the increase in liquid surface tension. The lower initial meniscus height has a smaller critical meniscus area. In addition, the rate increase in 
critical meniscus area is also smaller for a lower initial meniscus height. This conforms the observation made previously. These observations indicate that viscous force may be likely to take a dominate role if initial meniscus height is smaller.

Fig. 7 shows the effects of initial meniscus height and viscosity to the critical meniscus area in a dimensionless analysis.

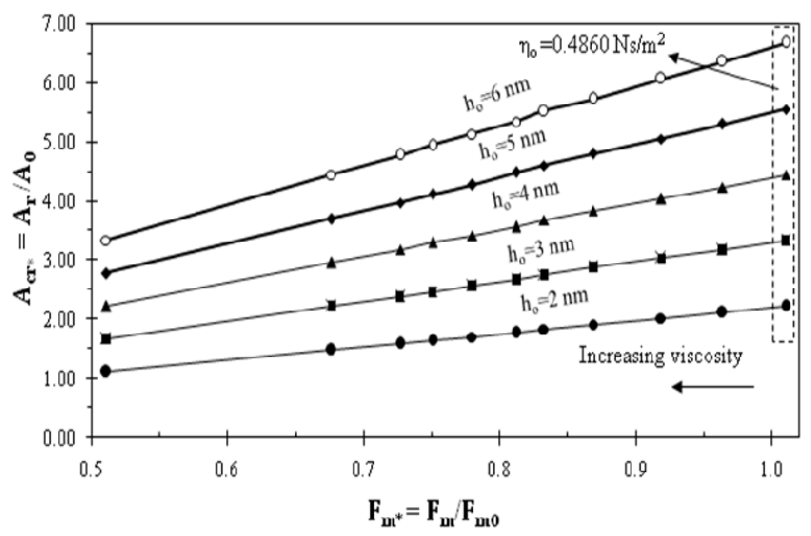

Fig. 7. Dimensionless meniscus force and critical meniscus area with contact angles $\Theta_{1}=\Theta_{2}=60^{\circ}$, constant $\gamma(0.0633 \mathrm{~N} / \mathrm{m})$ for separation time $=1 \mathrm{~s}$ for various viscosity $\left(\eta_{\mathrm{o}}=0.4860 \mathrm{Ns} / \mathrm{m}^{2}\right.$ (Silicon Oil))

In the analysis, the critical meniscus area was normalized to the initial meniscus area; and the dimensionless meniscus force was normalized to the initial static meniscus force. From the results, it is observed that the critical meniscus area, the critical meniscus area decreases with the increase in liquid viscosity for the cases the surface tension maintaining constant for a constant initial meniscus height. This is due to the more rapid increase in viscous force when the viscosity of the liquid is higher. Thus, the viscous force can dominate at smaller critical meniscus area. It is also noticed that the lower initial meniscus height has a smaller critical meniscus area. In addition, the rate decrease in critical meniscus area is also smaller for a lower initial meniscus height when the liquid viscosity is increased. These are similar to that observed in Fig. 6. The difference between the case of change in surface tension and change in viscosity is that the critical meniscus area increases with surface tension but decreases with viscosity.

From Fig. 6 and Fig. 7, a new observation can also be identified. The relationship between the critical meniscus area and the forces exhibits a nonlinear relationship when the surface tension of the liquid changes. For the change in viscosity, the relationship between the critical meniscus area and the forces exhibits a linear-like relationship.

\section{CONCLUSION AND SUGGESTION}

The analyses show that both meniscus force and viscous force are largely dependent on the separation distance. Both forces exhibit a rapid changing rate at the beginning. Meniscus force decreases with separation while viscous force has an opposite trend. The value of initial separation plays an important role affecting the break point and the magnitudes of the two forces.

Meniscus area significantly affects the magnitudes of the meniscus and viscous forces. Viscous force increases (with increase meniscus area) much faster as compared to meniscus force. The increase or decrease in the meniscus area can change the roles the two types of forces. For a fixed separation time $t_{s}$, the two types of forces are comparable when a critical meniscus area is reached. Larger or smaller value of the meniscus area leads to either viscous or meniscus force to be the dominant force of the system.

Initial meniscus height affects the role of the meniscus and viscous forces. The increase in the meniscus height leads to both meniscus force and viscous force decrease. Viscous force becomes dominant at relatively larger meniscus area when the initial meniscus height is larger.

Critical meniscus area (at which meniscus and viscous forces switch roles) changes with not only the initial meniscus height but also the liquid properties. Initially lower meniscus height with larger liquid viscosity leads to smaller critical meniscus area. Thus the viscous force can be a dominant force even though the liquid meniscus is very small.

When control of interfacial adhesion (due to liquid mediates contacting solid surfaces) is needed, the roles of the involved meniscus and viscous forces need to be determined. To effectively control the adhesion, the factors such as the initial separation height (of the two surfaces), the type of liquid, the formed meniscus area etc. all need to be considered.

\section{REFERENCES}

[1] S. Cai and B. Bhushan, "Meniscus and viscous forces during normal separation of liquid-mediated contacts," Nanotechnology, 465704465704, 2007

[2] S. Cai and B. Bhushan. "Meniscus and Viscous Forces during Separation of Hydrophilic and Hydrophobic Smooth/rough Surfaces with Symmetric and Asymmetric Contact Angles." Philos. Trans. A Math. Phys. Eng. Sci. vol. 366, no. 1870, pp. 1627-1647, 2008 a.

[3] S. Cai and B. Bhushan, "Meniscus and viscous forces during separation of hydrophilic and hydrophobic surfaces with liquid-mediated contacts." Mater. Sci. Eng. R-reports, vol. 61, no. 1-6, pp. 78-106, 2008b.

[4] F.M. Orr, L.E. Scriven, and A.P. Rivas, Pendular rings between solids: meniscus properties and capillary force, J. Fluid Mech., 67 (1975) 723742.

[5] H.W. Kang, H.J. Sung, T.M. Lee, D.S. Kim, and C.J. Kim, "Liquid Transfer between Two Separating Plates for Micro-gravure offset Printing,"J. Micromech. Microeng. vol. 19, pp. 015025, 2009.

[6] A.W. Adamson, Physical Chemistry of Surfaces, fifth ed., Wiley, New York, 1990.

[7] T. Stifter, O. Marti, and B. Bhushan, "Theoretical investigation of the distance dependence of capillary and van der waals forces in scanning force microscopy," Phys. Rev. B, vol. 62, pp. 667-673, 2000.

[8] B. Bhushan, Introduction to Tribology, John Wiley, New York, 2002.

[9] L.M. Hocking, The effect of slip on the motion of a sphere close to a wall and of two adjacent spheres, J. Eng. Maths., vol. 7, pp. 207-218, 1973. 